\title{
Input/output Linearization Control Technique for Anaerobic Digestion Reactor with Recirculation
}

\author{
Sura Srisuddee ${ }^{1}$, Malinee Sriariyanun ${ }^{1}$, Chanin Panjapornpon ${ }^{2}$, and Atthasit Tawai ${ }^{1, *}$ \\ ${ }^{1}$ Chemical and Process Engineering Program, The Siringhorn International Thai-German Graduate School of Engineering, King \\ Mongkut's University of Technology North Bangkok, Bangkok, Thailand \\ ${ }^{2}$ Department of Chemical Engineering, Faculty of Engineering, Kasetsart University, Bangkok, Thailand
}

\begin{abstract}
Anaerobic digestion (AD) process has been generally applied in factories for wastewater treatment and energy recovery. For the AD processes, the continuously stirred tank reactor (CSTR) with recirculation are typically applied in industries for methane production from wastewater treatment. Since the recycle stream affects the inlet concentration of the reactor, the control performance of traditional PI and PID controllers used to manipulate control actuators may be limited. Additionally, the process control loops related to biochemical reactions are generally employed the operational experiences without process dynamic consideration. A control system based on input/output (I/O) linearization control technique for an anaerobic digestion (AD) process was developed in this work. The control system applied the concept of the I/O linearization technique, which followed dynamic behaviors of the reactor with a two-step (acidogenesis-methanogenesis) kinetic model. The volatile fatty acid (VFA) concentration was regulated by manipulation of the dilution rate to achieve the requested trajectories. Control performances of the closed-loop system were investigated by a simulation under servo and regulatory problems. The simulation results showed that the developed control scheme successfully forced the controlled output to achieve the desired set points and handled the introduced control problems.
\end{abstract}

\section{Introduction}

Nowadays, wastewater is an important problem to be handled in industries. An alternative way to promote a greener production and solve this problem is anaerobic digestion (AD) process. The $\mathrm{AD}$ process has been generally applied for wastewater treatment and energy recovery because it has ability to transform the organic matter into biomethane [1]. The application of the AD process allow us to obtain benefits, compared with traditional aerobic treatment: low sludge production, little energy requirement, minimal safety requirement [2]. Normally, the AD process deals with two main biochemical reactions to produce biogas, acidogenesis and methanogenesis. The organic substrate, which normally characterized by chemical oxygen demand (COD), is consumed by acidogenic bacteria and converted into volatile fatty acids (VFA). After that, VFA will be used as substrate of methanogenic bacteria for the cell growth and biogas production. As mentioned that the $\mathrm{AD}$ process is a complex biochemical reaction process that presents the uncertain and nonlinear behaviors, process control systems that can handle the nonlinear dynamic behaviors are required. Therefore, a good design, analysis and evaluation of efficiency of the control systems are needed for the AD processes.

However, the traditional PI (proportional-integral) or PID (proportional-integral-derivative) controller applied to the $\mathrm{AD}$ processes are generally employed the operational experiences without process dynamic consideration. Moreover, the recirculation of the AD process can strongly affect the inlet concentration that need to be considered in the control system development. The advanced control systems such input/output (I/O) linearization technique have been applied for chemical processes to regulate the process with nonlinear behaviors. The process models, containing the kinetic expressions, are required to formulate the model-based control systems. Since the aim of this work was to design a control strucuture by applying the process model, the governing equations of the process need to be investigated. An example of AD process in the continuous stirred tank reactor (CSTR) system was presented in [2]. The dynamic models composed of two main biochemical reactions with a consideration of the total alkalinity and the total inorganic carbon in water. The growth rate of acidogenic bacteria was presented by Monod kinetics' model, while that of methanogenic bacteria was presented by Haldane kinetics' model. There was a number of research works proposed the control system for the AD processes. Zarrad et al. [3] proposed a linear quadratic (LQ) model to regulate the output at desired setpoint by manipulation of activated sludge and compared the control performance with the PI controllers. Mu et al. [4] proposed a multivariable linear quadratic tracking (LQT) approach by using by-pass and recycle stream to control the COD concentration in an upflow anaerobic sludge

\footnotetext{
*orresponding author: atthasit.t@tggs.kmutnb.ac.th
} 
blanket (UASB) reactor. The controlled complex reaction of $\mathrm{AD}$ process, which described by ODE system, was the challenging point in this work. Recently, the analytical model predictive control (AMPC) based on the $\mathrm{I} / \mathrm{O}$ linearization technique was applied for a UASB reactor described by an PDE-ODE model [5,6].

This work proposed an alternative control method for the AD process with a recycle stream. To formulate a control structure, the I/O linearization technique was applied to regulate the process variable at the desired setpoints. The main objective was to control the VFA concentration by manipulation of the dilution rate to achieve the desired target. An integral action was combined as a part of the control system to eliminate the mismatch of process and model. This developed control system was then applied to the wastewater treatment process for the methane production. In section 2, preliminaries of the mathematic models and $\mathrm{I} / \mathrm{O}$ linearization control definition were explained in this section. The description and mathematic modelling of AD process were presented in section 3 . The formulation of the control system was presented in section 4 . The simulation results, which were evaluated with servo and regulatory test were shown in section 5 . And the last section was conclusion that will be shown in section 6 .

\section{Prilimnaries}

\subsection{Problem formulation}

The system in equation (1) expressed the mathematical model of a CSTR with recirculation for the AD process. The state variables were shown in the metrix as follow :

$$
\begin{aligned}
\frac{\partial \xi}{\partial t} & =F-D \xi+K \mathbf{r}(\xi)-Q \\
y & =h(\xi)
\end{aligned}
$$

with the initial conditions

$$
\xi^{0}(0)=\xi_{\text {in }}
$$

where

$$
\begin{aligned}
\boldsymbol{\xi} & =\left[\begin{array}{l}
X_{1} \\
X_{2} \\
Z \\
S_{1} \\
S_{2} \\
C
\end{array}\right], \mathbf{r}(\xi)=\left[\begin{array}{l}
\mu_{1}(\xi) X_{1} \\
\mu_{2}(\xi) X_{2}
\end{array}\right], \mathbf{K}=\left[\begin{array}{ll}
1 & 0 \\
0 & 1 \\
0 & 0 \\
-k_{1} & 0 \\
k_{2} & -k_{3} \\
k_{4} & k_{5}
\end{array}\right] \\
\mathbf{D} & =\left[\begin{array}{llllll}
D & 0 & 0 & 0 & 0 & 0 \\
0 & D & 0 & 0 & 0 & 0 \\
0 & 0 & D & 0 & 0 & 0 \\
0 & 0 & 0 & D & 0 & 0 \\
0 & 0 & 0 & 0 & D & 0 \\
0 & 0 & 0 & 0 & 0 & D
\end{array}\right],
\end{aligned}
$$

$$
F=\left[\begin{array}{c}
0 \\
0 \\
D Z_{\text {in }} \\
D S_{1 i n} \\
D S_{2 i n} \\
D C_{\text {in }}
\end{array}\right], Q=\left[\begin{array}{c}
0 \\
0 \\
0 \\
0 \\
0 \\
q_{C}(\zeta)
\end{array}\right] \text { and } \xi_{\text {in }}=\left[\begin{array}{c}
X_{1}^{0} \\
X_{2}^{0} \\
Z^{0} \\
S_{1}^{0} \\
S_{2}^{0} \\
C^{0}
\end{array}\right]
$$

where $\xi$ denotes the vector of state variables, $\xi_{\text {in }}$ is the the vector of variables of inlet stream, $D$ and $Q$ are the dilution rate and the gas flow rate of the reactor. $F$ denotes the feed of reactor, $\mathbf{K}$ is a vector of the yield for substrate consumption or product formation. $\mathbf{r}(\xi)$ is the reaction rate of the reactor, $\mathbf{y}=\left[y_{1}, \ldots, y_{m}\right]^{T}$ denote the vector of controlled outputs, $\mathbf{t} \in[0, \infty]$ is the time and $h$ is a nonlinear function.

The model in (1) was later used to investigate the process responses and to formulate a control strategy for the CSTR reactor with recirculation.

\subsection{Input/output linearization control definition}

Consider the nonlinear system as the following form:

$$
\dot{\xi}=f(\xi, \mathbf{u}), y=h(\xi)
$$

where $\mathbf{u}$ denotes the vector of manipulated yariables, $\mathbf{y}$ denotes the vector of output variables and $\mathbf{f}$ is vector of a nonlinear function. The relative order of the nonlinear system in equation (2) is denoted by $r_{l}, \ldots, r_{m-1}, r_{m}$ where $r_{i}$ is the smallest integer [7] that $\partial\left[d^{r_{i}} y_{i} / d t^{r_{i}}\right] / \partial u \neq 0$ as expressed by the following equation :

$$
\begin{aligned}
& y_{i}=h(\xi) \\
& \frac{d y_{i}}{d t}=\left[\frac{\partial h}{\partial \xi} \frac{\partial \xi}{\partial t}\right]=h^{1}(\xi) \\
& \frac{d^{2} y_{i}}{d t^{2}}=\left[\frac{\partial h^{1}}{\partial \xi} \frac{\partial \xi}{\partial t}\right]=h^{2}(\xi) \\
& \vdots \\
& \frac{d^{r-1} y_{i}}{d t^{r-1}}=\left[\frac{\partial h^{r-2}}{\partial \xi} \frac{\partial \xi}{\partial t}\right]=h^{r-1}(\xi) \\
& \frac{d^{r} y_{i}}{d t^{r}}=\left[\frac{\partial h^{r-1}}{\partial \xi} \frac{\partial \xi}{\partial t}\right]=h^{r}(\xi, u)
\end{aligned}
$$

The closed-loop responses of the process state variables were requested as the following linear form:

$$
\left(\beta_{i} D+1\right)^{r_{i}}=\mathbf{v}_{\mathbf{i}}
$$

where $D$ is the differential operator (i.e. $D=d / d t$ ), $\mathbf{v}_{\mathbf{i}}$ is the compensated setpoint vectors and $\beta_{i}$ is the tuning 
parameter that can adjust the speed of the output response.

In order to formulate the controller, equation (3) and (4) are applied. The manipulated input $\mathbf{u}$ can be written in the compact form as:

$$
u(t)=\psi(\bar{\xi}, \mathbf{v})
$$

where $\bar{\xi}$ denotes the vector of the measured state variable.

\section{Description and mathematic modeling of anaerobic digestion process}

The biological reactions proposed by Bernard et al. [1] for macroscopic system of the anaerobic process can be used to explain the general biological pathways. The two main pathways, acidogenesis and methanogenesis, in the homogeneous continuous stirred tank reactor (CSTR) can be shown as follow:

- Acidogenesis reaction with reaction rate $r_{l}=\mu_{1} X_{l}$

$$
k_{1} S_{1} \stackrel{r_{1}}{\longrightarrow} X_{1}+k_{2} S_{2}+k_{4} C O_{2}
$$

- Methanogenesis reaction with reaction rate $r_{2}=\mu_{2} X_{2}$

$$
k_{3} S_{2} \stackrel{r_{2}}{\longrightarrow} X_{2}+k_{5} \mathrm{CO}_{2}+k_{6} \mathrm{CH}_{4}
$$

where $S_{I}$ represents the organic substrate characterized by COD. $S_{2}$ is shown the total concentration of VFA. $\mu_{1}$ and $\mu_{2}$ are shown the specific growth rates of acidogenesis and methanogenesis bacteria, respectively.

A schematic of anaerobic digestion process with a recycle stream was shown in Fig. 1 It was assumed that the $\mathrm{AD}$ process containd 2 reaction steps and the bacteria can be divided into two different groups with homogeneous characteristics.

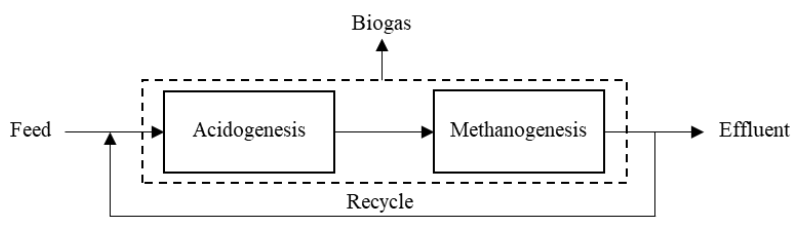

Fig. 1. One stage of anaerobic digestion process

For the acidogenesis, the organic substrate $\left(S_{l}\right)$, characterized by COD, was consumed by acidogenic bacteria $\left(X_{I}\right)$ to formulate the VFAs $\left(S_{2}\right)$ and carbon dioxide as the products. For the methanogenesis, the methanogenic bacteria $\left(X_{2}\right)$ utilized the VFAs as the substrates to produce methane and carbon dioxide gases. Introduction of the $\mathrm{AD}$ process in the CSTR system, the governing equations from material balances that applied the process model of [2] was shown as follow:

$$
\begin{aligned}
\frac{d X_{1}}{d t} & =D \cdot\left(X_{1}^{0}-X_{1}\right)+Y_{1} \cdot \mu_{1}\left(S_{1}\right) \cdot X_{1} \\
\frac{d X_{2}}{d t} & =D \cdot\left(X_{2}^{0}-X_{2}\right)+Y_{2} \cdot \mu_{2}\left(S_{2}\right) \cdot X_{2} \\
\frac{d S_{1}}{d t} & =D \cdot\left(\mathrm{S}_{1}^{0}-S_{1}\right)-\mu_{1}\left(S_{1}\right) \cdot X_{1} \\
\frac{d S_{2}}{d t}=D \cdot\left(\mathrm{S}_{2}^{0}-S_{2}\right)+c \cdot \mu_{1}\left(S_{1}\right) \cdot X_{1}-\mu_{2}\left(S_{2}\right) \cdot X_{2} & \frac{d Z}{d t}=D \cdot\left(Z_{0}-Z\right) \\
\frac{d C}{d t}= & D \cdot\left(C_{0}-C\right)-\mathrm{q}_{c}\left(S_{2}, Z, C\right)+Y_{3} \cdot \mu_{1}\left(S_{1}\right) \cdot X_{1} \\
& +Y_{4} \cdot \mu_{2}\left(S_{2}\right) \cdot X_{2}
\end{aligned}
$$

where $X_{1}, X_{2}, \quad S_{1}, \quad S_{2}$ are acidogenic bacteria, methanogenic bacteria, organic substrate and VFAs, respectively. The total alkalinity is shown by $Z$ and the total inorganic carbon in water which consist of dissolved carbon dioxide, bicarbonate and carbonate is shown by $C$.

$X_{0}^{1}, X_{0}^{2}, S_{0}^{1}, S_{0}^{2}, C_{0}$ and $Z_{0}$ are inlet concentrations which are affected by the recycle stream and $D(D=F / V)$ is dilution rate, The Monod kinetics for growths of acidogenic bacteria $\left(\mu_{1}\right)$ and Haldane kinetics for methanogenesis bacteria $\left(\mu_{2}\right)$ are shown as follow:

$$
\begin{aligned}
& \mu_{1}\left(S_{1}\right)=\frac{\mu_{1 \max } \cdot S_{1}}{Y_{1} \cdot\left(K_{S 1}+S_{1}\right)} \\
& \mu_{2}\left(S_{2}\right)=\frac{\mu_{2 \max } \cdot S_{2}}{Y_{2} \cdot\left(K_{S 2}+S_{2}+\frac{S_{2}^{2}}{K_{I}}\right)}
\end{aligned}
$$

where $\mu_{\text {Imax }}$ and $\mu_{2 \max }$ show the maximum growth rate of the two groups of bacteria, $K_{s I}$ is the saturation constant related with the organic substrate, and $K_{s 2}$ and $K_{i 2}$ are the saturation and inhibition constant related with the VFAs, respectively. The model parameters were listed in Table 1.

Table 1. Parameters of the AD process

\begin{tabular}{|l|l|l|}
\hline Symbol & \multicolumn{1}{|c|}{ Parameter } & \multicolumn{1}{c|}{ Value } \\
\hline$\mu_{\text {Imax }}$ & $\begin{array}{l}\text { Maximum acidogenic biomass } \\
\text { growth rate }\end{array}$ & $4.2 \mathrm{~d}^{-1}$ \\
\hline$\mu_{2 \max }$ & $\begin{array}{l}\text { Maximum methanogenic } \\
\text { biomass growth rate }\end{array}$ & $0.36 \mathrm{~d}^{-1}$ \\
\hline$K_{s 1}$ & Half-saturation constant for $\mathrm{S}_{1}$ & $0.0023 \mathrm{~g} / \mathrm{L}$ \\
\hline$K_{s 2}$ & Half-saturation constant for $\mathrm{S}_{2}$ & $2.3 \mathrm{mmol} / \mathrm{L}$ \\
\hline$K_{I 2}$ & Inhibition constant for $\mathrm{S}_{2}$ & $66.67 \mathrm{mmol} / \mathrm{L}$ \\
\hline$y_{1}, y_{2}$, & Yield coefficients & $0.11,0.00236$, \\
$y_{3,} y_{4}$ & $\begin{array}{l}\text { Stoichiometric coefficient of } \\
\text { the conversion of S1 to } \mathrm{S}_{2}\end{array}$ & $16.95 \mathrm{mmol} / \mathrm{g}$ \\
\hline$c$ & \multicolumn{2}{|l}{} \\
\hline
\end{tabular}


At the outlet of $\mathrm{AD}$ process, there were two mixed gases which were $\mathrm{CO}_{2}$ and $\mathrm{CH}_{4}$. The outlet molar of $\mathrm{CH}_{4}$ flow rate $\left(q_{m}\right)$ can be calculated by

$$
q_{m}=Y_{m} \mu_{2}\left(S_{2}\right) X_{2}
$$

And the outlet molar of $\mathrm{CO}_{2}$ flow rate $\left(q_{c}\right)$ can be calculated by

$$
q_{c}=k_{L} a\left[C+S_{2}-Z-K_{H} P_{C}\right]
$$

where the partial pressure of carbon dioxide gas was shown by $P_{c}$ that can be calculated by

$$
P_{c}=\frac{\varnothing-\sqrt{\varnothing^{2}-4 K_{H} P_{T}\left(C+S_{2}-Z\right)}}{2 K_{H}}
$$

with

$$
\varnothing=C+S_{2}-Z+K_{H} P_{T}+\frac{Y_{m}}{Y_{2} k_{L} a} \mu_{2}\left(S_{2}\right) X_{2}
$$

where $K_{h}, P_{t}$ and $k_{L} a$ showed the Henry's constant, total pressure and liquid/gas transfer rate, respectively. A block flow diagram of Fig. 2 presented the CSTR system with the process model.

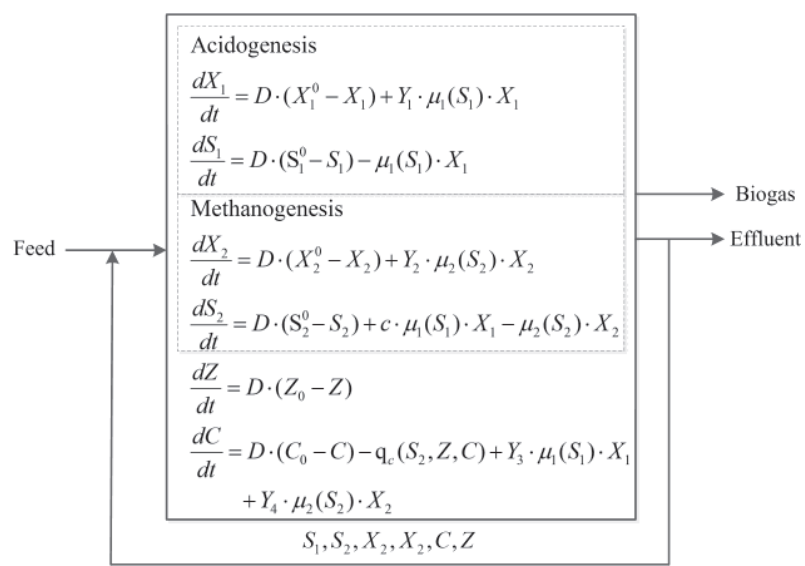

Fig. 2. A diagram of AD model in the CSTR system

The state variables of this model involved the substrates and microorganism that directly affected on $\mathrm{CH}_{4}$ and $\mathrm{CO}_{2}$ production, while total alkalinity and inorganic carbon affected on the gas-liquid equilibrium.

\section{Formulation of control system for the AD process}

In this study, the control objective was to regulate effluent VFA concentration of the CSTR with recycle stream at the desired set point by manipulating the dilution rate $(D)$. From the material balance equation, it was shown that the dilution rate affected on both COD $\left(S_{I}\right)$ and VFA $\left(S_{2}\right)$, which were the time-varying parameters and related to the methane production. The control structure of the proposed control system was shown in Fig. 3 The control system consisted of an I/O linearizing controller and a compensator, which expressed as an integral action.

\subsection{Input/output linearizing controller}

To develop the I/O linearizing control, the definition of relative order of (3) was applied. The control output, the VFA concentration, which was represented by $S_{2}$ was considered as a lumped variable. The controlled output can be shown in linearizing form as

$$
\beta D S_{2}+S_{2}=\mathbf{v}
$$

where $\beta$ is a tuning parameter [8]. By an application of the process model of equation (4) in (12), the equation of the control system can be solved. The compact form for the feedback control can be shown as

$$
u(t)=\psi\left(S_{2}, \mathbf{v}, \beta\right)
$$

This proposed controller was used to calculate the control action for each time step.

\subsection{Integral action}

For this proposed controller, the integral action was applied as a compensator and combined to the controller system. The compensator was used to handle the mismatch between the process and mathematical model. The equation of the proposed integral action was shown as

$$
\begin{aligned}
& \dot{\eta}=\lambda\left(y-y_{s p}\right) \\
& \mathbf{v}=\mathrm{S}_{2, s p}-\eta
\end{aligned}
$$

where $\eta$ is an integral action. The proposed $\mathrm{I} / \mathrm{O}$ linearizing controller of equation (12) was combined with the integral action for the control system development.



Fig. 3. The control structure of the proposed control system. 


\section{Simulation results}

\subsection{Closed-loop responses}

In this work, the nonlinear dynamic model of the CSTR reactor was simulated to investigate the process responses under the closed-loop system of the developed control strategy. The computer software such MATLAB was applied. To promote the VFA formation by the acidogenic bacteria, a minimum dilution rate of the reactor was set to be $0.15 \mathrm{~d}^{-1}$ at the beginning of the operation. Then the VFA $\left(S_{2}\right)$ was controlled by manipulating the dilution rate $(D)$ that contain a recycle stream with $R=10 \%$. The feed stream was mixed with the recycle stream before feeding to the reactor, the inlet concentration was continously updated during the process operation. The control performance of the proposed control scheme was compared to the PI controller with $K_{p}=0.12, K_{I}=0.15$. The initial inlet concentration of the reactor are $S_{1}^{0}=20 \mathrm{~g} / \mathrm{L}, S_{2}^{0}=40$ $\mathrm{mmol} / \mathrm{L}, X_{1}^{0}=0.1 \mathrm{~g} / \mathrm{L}, X_{2}^{0}=0.58 \mathrm{~g} / \mathrm{L}, Z_{0}=62 \mathrm{mmol} / \mathrm{L}$ and $C_{0}=65 \mathrm{mmol} / \mathrm{L}$. The tuning parameters of the $\mathrm{I} / \mathrm{O}$ linearization based control system were $\beta=0.4$ and $\lambda=0.15$. The simulation results of the closed-loop system of the developed control strategy were compared to the traditional PI controller. Figures 4 and 5 showed the closed-loop responses of the controlled output (S2) and the manipulated input variable (D), respectively.

The results showed that both control systems successfully force the controlled output at the desired set point. For the proposed control, the output achieved to the desired setpoint faster and presented less oscillation than PI controller. Although, the inlet concentration affected by the recycle stream led to the fluctuation of the output stream concentration. It can be seen from the process responses that the control systems attempted to manipulate the output around the required target.

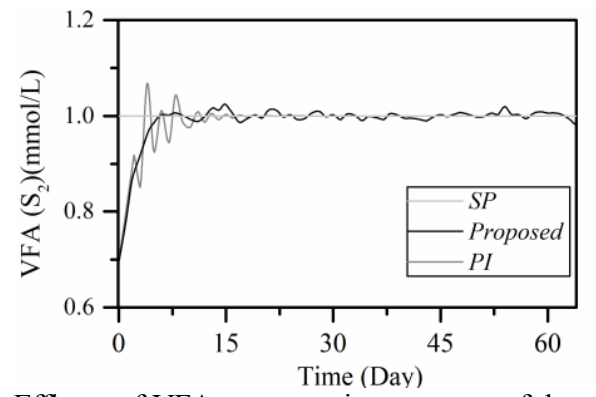

Fig. 4. Effluent of VFA concentration response of the closed-loop system.

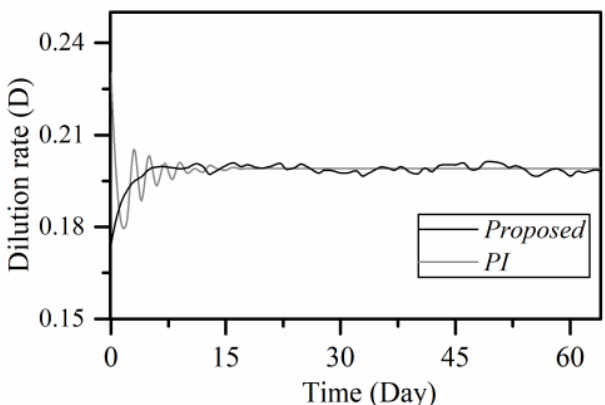

Fig. 5. Dilution rate corresponding to the closed-loop system of Fig. 4.

\subsection{Control performance}

The control performance of the developed control system was evaluated by introducing the servo and regulatory problems. For the servo test, it was applied when the control output achieved the desired set point. From the initial set point, which was set at $1 \mathrm{mmol} / \mathrm{L}$, then it was adjusted to be $2.5 \mathrm{mmol} / \mathrm{L}$. The dilution rate of the reactor was adapted by the proposed controlle to force the control output to the new set point (Fig. 6 and 7 ,). The results showed that the developed controller can regulate the control output to follow the desired conditions asymptotically. For the PI controller, it presented the aggressive adjustment of the manipulated input, which caused strong fluctuations of the output.

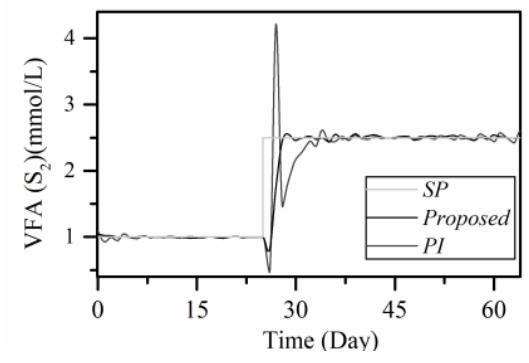

Fig. 6. Closed-loop response of the effluent of VFA concentration under servo test.

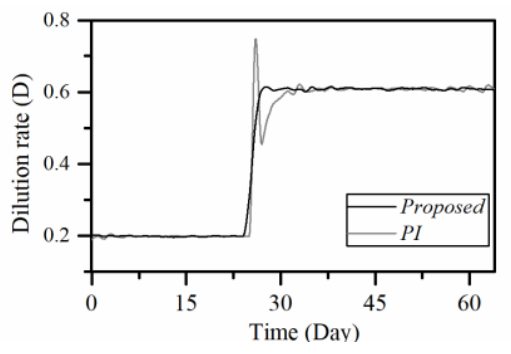

Fig. 7 Dilution rate corresponding to the servo test.

For achieving the set point, the controller adjusted the dilution rate to balance the substrate for the microorganisms. The results of regulatory tests showed that the proposed control system had a higher capability in handling the regulatory problem compared to the PI controller (Fig. 8 and 9). It was clear that the developed control scheme, which applied the biochemical reaction model to formulate the controller based on the I/O linearization technique with a compensator, successfully forced the process to the desired set points and stabilize the system under the condition with disturbances.

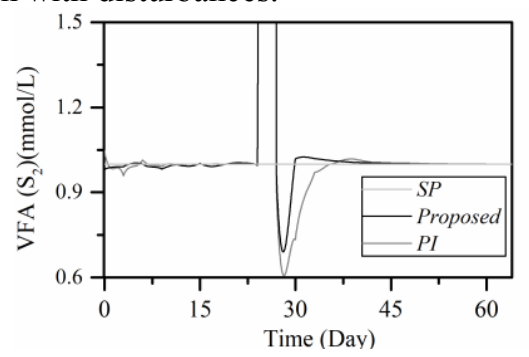

Fig. 8. Closed-loop response of the effluent of VFA concentration under regulatory test.

\footnotetext{
* Corresponding author: atthasit.t@tggs.kmutnb.ac.th
} 


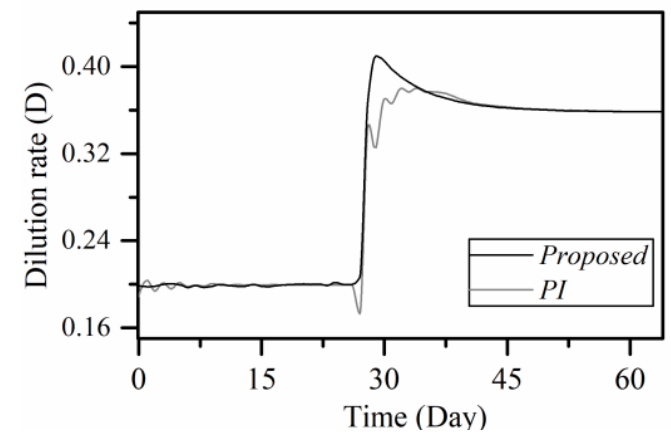

Fig. 9. Dilution rate corresponding to the regulatory test

\section{Conclusion}

The development of a control system based on the feedback I/O linearization technique for an $\mathrm{AD}$ process with recirculation was proposed in this study. To handle the nonlinear behaviors of the reactor with biochemical reactions and the effects of the recycle stream, the controller applied the kinetic models with an updated mixed stream concentration. Since methane production was related to the VFA concentration, the control objective was focused on the regulation of the effluent VFA concentration at the desired setpoint by manipulating the dilution rate. The controller was combined with an integral action to provide the compensation of the process/model mismatch, and then the developed control scheme was applied to the process. Simulation results showed that the developed control system successfully forced the process to the desired targets and had a capability to stabilize the system under the condition with disturbances. In addition, the requirement of a few tuning parameters allow us to easily implement the proposed control strategy to the AD processes.

This research was funded by King Mongkut's University of Technology North Bangkok. Contract no. KMUTNB-62KNOW-10

\section{References}

1. O. Bernard, Z. Hadj-Sadok, D. Dochain, A. Genovesi, J.P. Steyer, Biotechnol. Bioeng. 75, 424438 (2001)

2. Z. Duan, MNC. Bournazou, C. Kravaris, Chem Eng J. 327, 1102-1116 (2017)

3. W. Zarrad, J. Harmand, M.Devisscher, J.P. Steyer, Control Eng. Pract. 12, 323-333 (2004)

4. S. Mu, Y. Zeng, P.Wu, J. Chem. Technol. Biotechnol. 83, 892-903 (2008)

5. A. Tawai, C. Panjapornpon, M. Sriariyanun, K. Cheenkachorn, IFAC-PapersOnLine. 51(28), 109114 (2018)

6. A. Tawai, C. Panjapornpon, P. Dittanet, Ind. Eng. Chem. Res. 55, 10121-10131 (2016)

7. P. Sanposh, W. Leenanithikul, S. Phoojaruenchanachai, C. Panjapornpon, ECTI-CON 2008. 2, 613-616 (2008)

8. W. Chonwattana, C. Panjapornpon, A. Tawai, Aided Chem. Eng. 108, 372-381 (2018) 